\title{
The Impact of Documentation Style on Influenza-Like IIIness Rates in the Emergency Department
}

\author{
Dino P. Rumoro', Shital C. Shah ${ }^{1}$, Gillian S. Gibbs ${ }^{\star 1}$, Marilyn M. Hallock', Gordon M. \\ Trenholme ${ }^{1}$, Michael J. Waddell ${ }^{2}$ and Joseph P. Bernstein ${ }^{3}$
}

${ }^{1}$ Rush University, Chicago, IL, USA; 'Pangaea Information Technologies, Ltd, Chicago, IL, USA; ${ }^{3} \mathrm{H}-\mathrm{CORE}, \mathrm{LLC}, \mathrm{Chicago}, \mathrm{IL}, \mathrm{USA}$

\section{Objective}

To compare the influenza-like illness (ILI) rates in the emergency departments (ED) of a community hospital versus a large academic medical center (AMC).

\section{Introduction}

In recent years, the threat of pandemic influenza has drawn extensive attention to the development and implementation of syndromic surveillance systems for early detection of ILI. Emergency department (ED) data are key components for syndromic surveillance systems. However, the lack of standardization for the content in chief complaint (CC) free-text fields may make it challenging to use these elements in syndromic surveillance systems. Furthermore, little is known regarding how ED data sources should be structured or combined to increase sensitivity without elevating false positives. In this study, we constructed two different models of ED data sources and evaluated the resulting ILI rates obtained in two different institutions.

\section{Methods}

Geographic Utilization of Artificial Intelligence in Real-Time for Disease Identification and Alert Notification (GUARDIAN) - a syndromic surveillance program - was used to automate ILI detection using chief complaints with free text and vital signs (i.e., ILI $_{\mathrm{v} 1}$ ), and the entire Electronic Medical Record (EMR) (i.e., $\mathrm{ILI}_{\mathrm{V} 2}$ ) for a large AMC and a community hospital during the 2014-2015 influenza season. The GUARDIAN system defined ILI as fever (temperature $\geq 100^{\circ} \mathrm{F}$ ) and cough and/or sore throat.

The first step in our data analysis was to compute the daily ILI rates (i.e., the total number of ILI patients divided by the total ED census per day) and the 7-day moving average ILI rates (hence forth referred as the ILI rate). Thereafter, we performed graphical and statistical (i.e., descriptive and ANOVA with post hoc Bonferroni test) analyses and compared the results generated from the data obtained through the two ILI detection approaches.

\section{Results}

Compared to the AMC ILI rate for ILI $_{\mathrm{v} 1}$, the community hospital had significantly higher ILI rates for both ILI $\mathrm{v}_{\mathrm{v} 1}$ and $\mathrm{ILI}_{\mathrm{v} 2}(\mathrm{p}<0.01)$, as shown in Table 1. For ILI $\mathrm{V}_{\mathrm{V} 1}$, the community hospital had, on average, $3.84 \%$ higher ILI rates as compared to the AMC. In addition, ILI ${ }_{\mathrm{V} 2}$ yielded higher mean ILI rates than ILI $_{\mathrm{V} 1}$ in both institutions, with a difference of $1.89 \%$ for the community hospital and $7.55 \%$ for the AMC. Figure 1 indicates that the ILI rates derived from $\operatorname{ILI}_{\mathrm{V} 1}$ and $\mathrm{ILI}_{\mathrm{V} 2}$ for the community hospital and the ILI rates derived from ILI for the AMC are typically above 5\%, while the ILI rates derived from $\mathrm{ILI}_{\mathrm{V} 1}$ for the AMC is below 5\%. All pairs of ILI models vs. settings were significantly different from each other $(\mathrm{F}=217.84, \mathrm{df}=949$, $\mathrm{p}<0.01)$.

\section{Conclusions}

The community hospital had higher $\operatorname{ILI}_{\mathrm{V} 1}$ rates as compared to the AMC mainly due to frequent use of CC free-text fields by community hospital triage nurses. There are clinical, operational, and cultural differences between AMC and community hospital EDs.
With detailed clinical documentation at triage, surveillance systems can generate reliable probabilities/weights for early ILI detection. Since CC free-text fields are so data rich, surveillance systems that utilize natural language processors can improve ILI and other disease detection. In addition, depending on the setting, institutions similar to the AMC in this study should consider using the entire EMR for ILI surveillance.

Table 1: Comparison of ILI models between AMC and community hospital settings

\begin{tabular}{|c|c|c|}
\hline \multirow[t]{2}{*}{ ILI Models } & \multicolumn{2}{|c|}{$\begin{array}{c}7 \text { Day Moving Average ILI Rates (Mean } \pm \text { Standard } \\
\text { Deviation) }\end{array}$} \\
\hline & AMC & Community Hospital \\
\hline $\begin{array}{c}\text { ILI }_{\mathrm{V} 1} \text { - Chief complaints with free text and } \\
\text { vital signs }\end{array}$ & $3.81 \% \pm 1.76 \%$ & $7.65 \% \pm 3.53 \%$ \\
\hline ILIV2 - Entire EMR & $11.36 \% \pm 3.08 \%$ & $9.54 \% \pm 4.34 \%$ \\
\hline
\end{tabular}

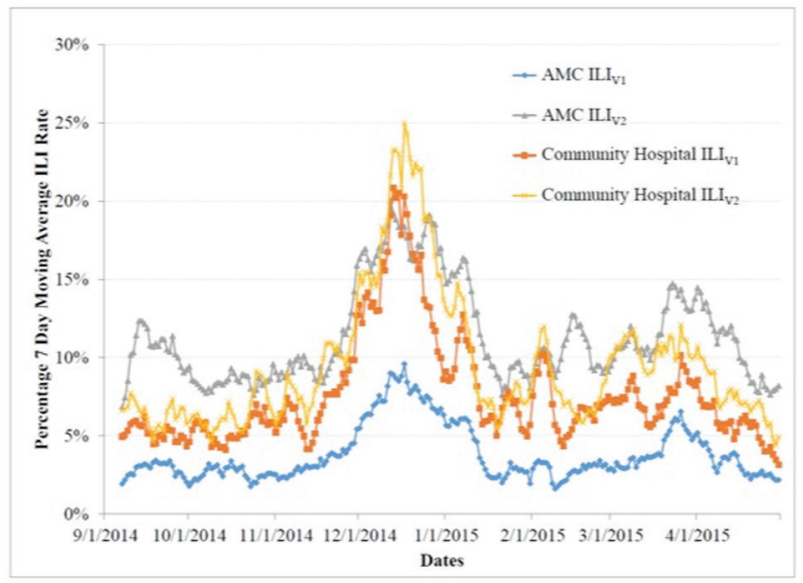

Figure 1. Comparison of ILI models between AMC and community hospital settings

\section{Keywords}

GUARDIAN; Influenza-like illness; Emergency department

\section{Acknowledgments}

GUARDIAN is funded by the US Department of Defense, Telemedicine and Advanced Technology Research Center, Award numbers W81XWH-09-1-0662 and W81XWH-11-1-0711.

\section{*Gillian S. Gibbs}

E-mail: Gillian_Gibbs@rush.edu 